\title{
Clinical significance of day 5 peripheral blast clearance rate in the evaluation of early treatment response and prognosis of patients with acute myeloid leukemia
}

Cong Yu ${ }^{\dagger}$, Qing-lei Kong ${ }^{\dagger}$, Yun-xiang Zhang ${ }^{\dagger}$, Xiang-qin Weng, Jing Wu, Yan Sheng, Chun-lei Jiang, Yong-mei Zhu, Qi Cao, Shu-min Xiong, Jun-min Li, Xiao-dong Xi, Sai-juan Chen ${ }^{*}$ and Bing Chen ${ }^{*}$

\begin{abstract}
Background: Minimal residual disease detection in the bone marrow is usually performed in patients with acute myeloid leukemia undergoing one course of induction chemotherapy. To optimize the chemotherapy strategies, more practical and sensitive markers are needed to monitor the early treatment response during induction. For instance, peripheral blood (PB) blast clearance rate may be considered as such a monitoring marker.
\end{abstract}

Methods: PB blasts were monitored through multiparameter flow cytometry (MFC). Absolute counts were determined before treatment $\left(D_{0}\right)$ and at specified time points of induction chemotherapy $\left(D_{3}, D_{5}, D_{7}\right.$, and $\left.D_{9}\right)$. The cut-off value of $D_{5}$ peripheral blast clearance rate (D5-PBCR) was defined through receiver operating characteristic (ROC) analysis. Prognostic effects were compared among different patient groups according to D5-PBCR cut-off value.

Results: D5-PBCR cut-off value was determined as $99.55 \%$. Prognostic analysis showed that patients with D5-PBCR $\geq 99.55 \%$ more likely achieved complete remission (94.6\% vs. $56.1 \%, P<0.001)$ and maintained a relapse-free status than other patients $(80.56 \%$ vs. $57.14 \%, P=0.027)$. Survival analysis revealed that relapse-free survival (RFS) and overall survival (OS) were longer in patients with D5-PBCR $\geq 99.55 \%$ than in other patients (two-year OS: $71.0 \%$ vs. 38.7\%, $P=0.011$; two-year RFS: $69.4 \%$ vs. $30.7 \%, P=0.026$ ). In cytogenetic-molecular intermediate-risk group, a subgroup with worse outcome could be distinguished on the basis of D5-PBCR (<99.55\%; OS: $P=0.033$, RFS: $P=0.086$ ).

Conclusions: An effective evaluation method of early treatment response was established by monitoring PB blasts through MFC. D5-PBCR cut-off value (99.55\%) can be a reliable reference to predict treatment response and outcome in early stages of chemotherapy. The proposed marker may be used in induction regimen modification and help optimize cytogenetic-molecular prognostic risk stratification.

Keywords: Peripheral blast clearance rate, Early treatment response, Prognosis, AML

\footnotetext{
*Correspondence: sjchen@stn.sh.cn; chenbing.rjsih@yahoo.com

${ }^{\dagger}$ Equal contributors

State Key Laboratory of Medical Genomics, Shanghai Institute of Hematology, Rui Jin Hospital, affiliated to Shanghai Jiao Tong University (SJTU) School of Medicine, Collaborative Innovation Center of Systems Biomedicine, SJTU, Shanghai, China
}

\section{Biomed Central}

(c) 2015 Yu et al.; licensee BioMed Central. This is an Open Access article distributed under the terms of the Creative Commons Attribution License (http://creativecommons.org/licenses/by/4.0), which permits unrestricted use, distribution, and reproduction in any medium, provided the original work is properly credited. The Creative Commons Public Domain Dedication waiver (http://creativecommons.org/publicdomain/zero/1.0/) applies to the data made available in this article unless otherwise stated. 


\section{Background}

Acute myeloid leukemia (AML) is a group of clinically and genetically heterogeneous diseases $[1,2]$. Despite treatment advancements in acute promyelocytic leukemia (M3), current treatment of AML is based on chemotherapy. Standard induction chemotherapy consists of anthracycline and cytarabine $(3+7$ regimen $)$ can achieve the complete remission (CR) rate of approximately $75 \%$, but outcome is uncertain because of the variability of individual genetic profile and drug sensitivity [3,4]. Intense chemotherapy or allogeneic hematopoietic cell transplantation (allo-HSCT) can benefit patients who are refractory or tend to relapse [5]. Early and easy monitoring of minimal residual disease (MRD) reflects treatment response in time and becomes an essential reference for patients with AML to optimize chemotherapy.

Multiparameter flow cytometry (MFC) has been used as a standard technique to track MRD in leukemia patients in the past decades [6]. In patients with acute lymphoblastic leukemia (ALL), the threshold of $0.01 \%$ of the bone marrow (BM) MRD is considered as the boundary of relapse predict index $[7,8]$. In AML, ambiguous threshold is approximately $0.1 \%$ and accuracy is approximately 10 times lower than that in ALL [9]. Moreover, BM MRD status in AML cannot be considered as an independent prognostic predictor, even though this status is considered as such in ALL [8].

Either in ALL or AML patients, the time point of BM MRD measurement is usually after $C R$, which may be late to determine early treatment response. Patients also hesitate to undergo frequent BM aspirations. Thus, peripheral blood (PB) blast clearance in early stage of chemotherapy has been extensively investigated. In ALL, encouraging results have been reported; for instance, the complete clearance of PB blasts within the first week of treatment may be related to $C R$ achievement $[10,11]$. In patients with AML, the $\mathrm{PB}$ blast clearance rate (PBCR) is closely correlated with treatment response and survival, but sampling time point and cut-off value vary [12-16].

In this study, we assessed the prognostic value of PBCR during induction in a cohort of 96 newly diagnosed AML patients. An earlier, easier, and more accurate technique than current systems has been established to distinguish high-risk patients and to enable a prompt improvement of induction chemotherapy.

\section{Results}

\section{Patient characteristics}

From June 2011 to August 2014, 96 newly diagnosed de novo AML (non-M3) were included in the study. Patient characteristics are summarized in Table 1. Median age was 44.5 years (14-74). Median WBC counts and circulating blasts were $13.3 \times 10^{9} / \mathrm{L}$ (range, $1.32 \times 10^{9} / \mathrm{L}$ to
$249.90 \times 10^{9} / \mathrm{L}$ ) and $3730.7 / \mu \mathrm{L}$ (range, $11.32 / \mu \mathrm{L}$ to $246,000 /$ $\mu \mathrm{L})$, respectively. The median percentage of BM blasts was $65 \%$ (14.5\% to $98 \%)$.

Leukemia-associated aberrant immunophenotypes (LAIPs) were identified in $72(75 \%)$ patients with four main types: 63 cases with cross-lineage antigen expression, 6 cases with asynchronous antigen expression, 13 cases with antigen $\mathrm{dim} /$ strong expression, and 8 cases with antigen expression missing. The details of LAIP distribution were listed in Additional file 1: Table S1.

Among the 58 cases with normal or unavailable karyotype, NRAS mutations were found in 4 cases (7.14\%), NPM1 in 12 (21.43\%), FLT3-ITD in 6 (10.71\%), FLT3-TKD in 2 (3.51), DNMT3A in 12 (20.69\%), CEBPA biallelic in 16 (28.07\%), MLL-PTD in 4 (7.02\%), and $M L L$-fusion gene was found in $2(3.51 \%)$ patients. According to the cytogenetic-molecular prognostic risk classification, 23, 53, and 20 patients were classified into favorable, intermediate, and unfavorable groups, respectively.

CR was achieved in $69.79 \%$ (67/96) of patients after one course of induction, and LAIPs could be detected in $77.61 \%(52 / 67)$ at diagnosis. After induction, 29 patients (55.77\%) remained MRD positive $(\geq 0.1 \%)$ by MFC. Total relapse rate was $32.5 \%(26 / 80)$.

\section{Relationship between treatment response and PB blast reduction ratio}

A rapid reduction in PB blasts was observed in the CR group vs. in the non-complete remission (NCR) group, with PB blast reduction ratios (PBRRs) of $1.09 \pm 0.62$ vs. $0.70 \pm 0.53,2.49 \pm 0.92$ vs. $1.70 \pm 0.70,3.41 \pm 1.02$ vs. $2.49 \pm 0.95$, and $3.92 \pm 1.28$ vs. $2.91 \pm 1.13$ on days 3 $\left(\mathrm{D}_{3}\right), 5\left(\mathrm{D}_{5}\right), 7\left(\mathrm{D}_{7}\right)$, and $9\left(\mathrm{D}_{9}\right)$, respectively $\left(\mathrm{D}_{3}: P=0.005\right.$, $\mathrm{D}_{5}: P<0.0001, \mathrm{D}_{7}: P<0.0001$, and $\mathrm{D}_{9}: P<0.0001$; Table 2).

In 52 patients who were under LAIP surveillance after $\mathrm{CR}$, no significant differences in PBRRs were observed between MRD-positive and negative groups (Table 2). Significantly higher PBRRs were observed in non-relapse vs. relapse group, with $2.59 \pm 0.93$ vs. $2.00 \pm 0.68,3.57 \pm$ 0.98 vs. $2.83 \pm 0.78$, and $4.16 \pm 1.19$ vs. $3.15 \pm 1.02$ on $\mathrm{D}_{5}, \mathrm{D}_{7}$, and $\mathrm{D}_{9}$, respectively $\left(\mathrm{D}_{5}: P=0.005, \mathrm{D}_{7}: P=\right.$ $\left.0.001, \mathrm{D}_{9}: P=0.0005\right)$. In patients who experienced relapse within 6 months (early relapse), significant differences in PBRRs on $D_{5}, D_{7}$, and $D_{9}$ were also observed (D $: 2.52 \pm 0.89$ vs. $1.91 \pm 0.80, P=0.017 ; D_{7}: 3.48 \pm 0.97$ vs. $2.76 \pm 0.81, P=0.008 ; \mathrm{D}_{9}: 4.02 \pm 1.19$ vs. $3.14 \pm 1.10$, $P=0.009$; Table 2).

\section{Determination of D5-PBCR cut-off value}

The PBRR of $\mathrm{D}_{5}$ showed the highest significant difference between early $\mathrm{CR}$ and NCR groups. The difference in PBRRs related to the relapse status also showed that $D_{5}$ was the first time point to appear with a definite 
Table 1 Clinical characteristics of all patients and stratified by D5 peripheral blast clearance rate (D5-PBCR)

\begin{tabular}{|c|c|c|c|c|}
\hline Characteristics & All patients $(n=96)^{a}$ & D5-PBCR $\geq 99.55 \%(n=37)$ & D5-PBCR $<99.55 \%(n=57)$ & $P$ value \\
\hline Age, y, median (range) & $44.5(14-74)$ & $44(15-67)$ & $45(14-74)$ & 0.467 \\
\hline Gender (no.) & & & & 0.996 \\
\hline Male & 61 & 24 & 37 & \\
\hline Female & 35 & 13 & 20 & \\
\hline WBC count $\left(\times 10^{9} / \mathrm{L}\right)$, median (range) & $13.3(1.32-249.90)$ & $26.99(3.2-120.9)$ & $9.20(1.32-249.90)$ & 0.160 \\
\hline Hemoglobin (g/L), median (range) & $85.0(34.90-136.00)$ & $89.5(55.0-136.0)$ & $82.0(34.9-125.0)$ & 0.051 \\
\hline Platelet $\left(\times 10^{9} / L\right)$, median (range) & $38.0(3.00-455.00)$ & $38.5(6.0-179.0)$ & $36.0(3.0-221.0)$ & 0.975 \\
\hline Blasts in bone marrow (\%), median (range) & $65.0(14.50-98.00)$ & $71.0(21.5-96)$ & $60.1(14.5-98.0)$ & 0.038 \\
\hline Blasts in peripheral blood $(/ \mu \mathrm{L})$, median (range) & $3730.7(11.32-246,000)$ & $4961.7(479.9-124,900)$ & $3730.7(11.32-246,000)$ & 0.035 \\
\hline FAB subtypes (no.) & & & & 0.229 \\
\hline M1 & 2 & 1 & 1 & \\
\hline M2 & 24 & 5 & 18 & \\
\hline M4 & 43 & 19 & 24 & \\
\hline M4Eo & 2 & 2 & 0 & \\
\hline M5 & 19 & 7 & 11 & \\
\hline M6 & 1 & 1 & 0 & \\
\hline NA & 5 & 2 & 3 & \\
\hline Cytogenetic-molecular risk group (no.) & & & & 0.045 \\
\hline Favorable & 23 & 12 & 11 & 0.148 \\
\hline Intermediate & 53 & 22 & 30 & 0.515 \\
\hline Unfavorable & 20 & 3 & 16 & 0.019 \\
\hline LAIP (no.) & & & & - \\
\hline Cross-lineage antigen expression & 63 & 29 & 34 & \\
\hline Asynchronous antigen expression & 6 & 0 & 6 & \\
\hline Antigen dim/strong expression & 13 & 6 & 6 & \\
\hline Antigen expression missing & 8 & 4 & 3 & \\
\hline CR (\%) & 67 (69.79) & 35 (94.6) & $32(56.1)$ & $<0.001$ \\
\hline $\operatorname{MRD}(+)(\%)^{\mathrm{b}}$ & $29(55.77)$ & $14(50)$ & 15 (62.5) & 0.366 \\
\hline Relapse (\%) & $26(32.50)$ & 7 (19.44) & $18(42.86)$ & 0.027 \\
\hline
\end{tabular}

$N A$ not available, $C R$ achieved complete remission after one course of induction therapy, LAIP leukemia-associated aberrant immunophenotype, FAB French-American-British, MRD minimal residual disease. ${ }^{a}$ Two patients lacking in the peripheral blast absolute counts data of day 5 could not be further stratified into different groups according

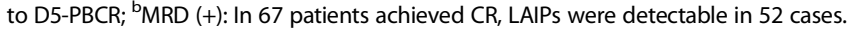

prognostic value. Receiver operating characteristic (ROC) analysis was performed to evaluate the predictive power of PBRR on patients' complete remission. The area under the curve (AUC) of $\mathrm{D}_{5}$ was 0.746 , which was larger than that of $\mathrm{D}_{7}(0.736)$ and $\mathrm{D}_{9}(0.719)$, and hence showed more statistical significance. Thus, $\mathrm{D}_{5}$ was chosen as the time point to determine D5 peripheral blast clearance rate (PBCR) cut-off value.

The day 5 PB blast reduction ratio (D5-PBRR) of 2.35 was selected as the optimal cut-off, according to the maximum sum of the sensitivity and specificity with $52.2 \%$ and $92.6 \%$ on the ROC curve, respectively (Figure 1 ). A logarithmic value of 2.35 of PBRR is equal to the clearance rate of $99.55 \%$ initial peripheral blasts; thus, D5-PBCR was determined as $99.55 \%$.

\section{Prognostic impact of D5-PBCR}

As shown in the three rightmost columns of Table 1, patients were divided into two groups according to the D5-PBCR cut-off value. The two groups' clinical characteristics, including age, gender, French-American-British (FAB) subtype, and initial WBC, were comparable. Patients with higher D5-PBCR $(\geq 99.55 \%)$ presented higher percentage of BM blasts $(71.0 \%$ vs. $60.1 \%, P=0.038)$, and higher $\mathrm{PB}$ blast counts $(4961.7 / \mu \mathrm{L}$ vs. $3730.7 / \mu \mathrm{L}$, $P=0.035)$ at diagnosis. 
Table 2 Correlation of PB blast reduction ratio (PBRR) with treatment response

\begin{tabular}{|c|c|c|c|c|}
\hline & \multicolumn{4}{|c|}{ PB blast reduction ratio $(\log 10)( \pm S D)$} \\
\hline & Day 3 & Day 5 & Day 7 & Day 9 \\
\hline $\operatorname{CR}(n=67)$ & $1.09 \pm 0.62$ & $2.49 \pm 0.92$ & $3.41 \pm 1.02$ & $3.92 \pm 1.28$ \\
\hline $\operatorname{NCR}(n=29)$ & $0.70 \pm 0.53$ & $1.70 \pm 0.70$ & $2.49 \pm 0.95$ & $2.91 \pm 1.13$ \\
\hline$P$ value & 0.005 & $<0.0001$ & $<0.0001$ & $<0.0001$ \\
\hline $\operatorname{MRD}(+) n=23)$ & $1.05 \pm 0.60$ & $2.43 \pm 1.05$ & $3.19 \pm 1.11$ & $3.62 \pm 1.41$ \\
\hline $\operatorname{MRD}(-)(n=29)$ & $1.25 \pm 0.64$ & $2.69 \pm 0.78$ & $3.19 \pm 1.11$ & $4.15 \pm 1.13$ \\
\hline$P$ value & 0.266 & 0.334 & 0.162 & 0.157 \\
\hline Relapse $(+)(n=25)$ & $0.85 \pm 0.45$ & $2.00 \pm 0.68$ & $2.83 \pm 0.78$ & $3.15 \pm 1.02$ \\
\hline Relapse $(-)(n=54)$ & $1.12 \pm 0.67$ & $2.59 \pm 0.93$ & $3.57 \pm 0.98$ & $4.16 \pm 1.19$ \\
\hline$P$ value & 0.066 & 0.005 & 0.001 & 0.0005 \\
\hline Early relapse $^{a}(+)(n=16)$ & $0.80 \pm 0.45$ & $1.91 \pm 0.80$ & $2.76 \pm 0.81$ & $3.14 \pm 1.10$ \\
\hline Early relapse $^{a}(-)(n=63)$ & $1.10 \pm 0.65$ & $2.52 \pm 0.89$ & $3.48 \pm 0.97$ & $4.02 \pm 1.19$ \\
\hline$P$ value & 0.094 & 0.017 & 0.008 & 0.009 \\
\hline
\end{tabular}

$C R$ achieved complete remission after one course of induction therapy, $N C R$ not achieved complete remission, MRD minimal residual disease. ${ }^{\mathrm{a} E a r l y}$ relapse: hematologic relapse within 6 months.

Patients with higher D5-PBCR $(\geq 99.55 \%)$ were more likely to achieve CR $(94.6 \%$ vs. $56.1 \%, P<0.001)$ and exhibit less relapse rate $(19.44 \%$ vs. $42.86 \%, P=0.027)$. However, post-induction BM MRD showed no significant difference between the two D5-PBCR groups (MRD positive: $50 \%$ vs. $62.5 \%, P=0.366$ ).

In molecular studies, a trend of more NRAS $(14.8 \%$ vs. $0 \%, P=0.051)$, CEBPA biallelic mutations $(37.0 \%$

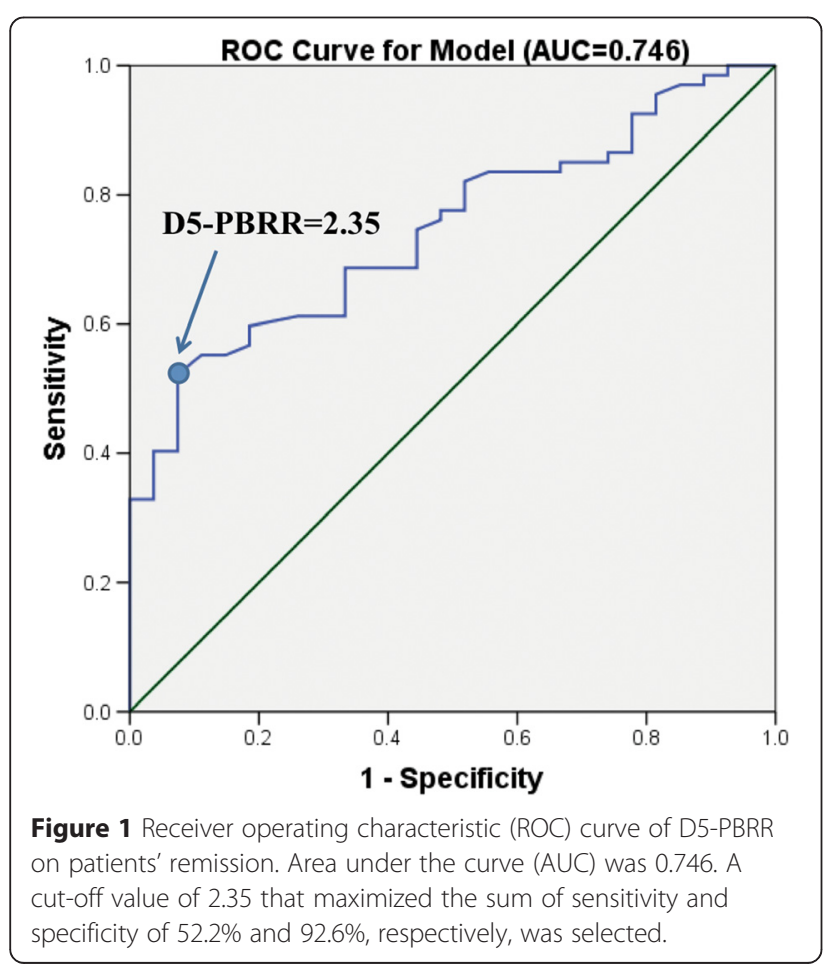

vs. $20.7 \%, P=0.142$ ), and less $M L L-P T D$ mutations (3.7\% vs. $10.3 \%, P=0.612$ ) were observed in the D5PBCR $\geq 99.55 \%$ group (Additional file 2: Table S2).

The distribution of the cytogenetic-molecular risk classification showed that more unfavorable cases were classified into the lower D5-PBCR (<99.55\%) group $(16 / 19, P=0.019)$. In the intermediate risk group, although the distribution was similar, patients with low D5-PBCR showed significant adverse survival [estimated two-year overall survival (OS): $25.6 \%$ vs. $76.4 \%$, $P=0.033$; estimated two-year relapse-free survival (RFS): $23.9 \%$ vs. $71.5 \%, P=0.086$ ] (Figure $2 \mathrm{~A}$ and $\mathrm{B}$ ), even close to that of the unfavorable-risk group (Additional file 3: Figures S1A and S1B). In the favorable-risk group, no differences were observed in distribution or survival analysis.

Further analysis of the whole cohort showed that higher D5-PBCR ( $\geq 99.55 \%$ ) was associated with significantly longer OS and RFS (estimated two-year OS: $71.0 \%$ vs. $38.7 \%$, $P=0.011$; estimated two-year RFS: $69.4 \%$ vs. $30.7 \%$, $P=0.026$; Figure $3 \mathrm{~A}$ and $\mathrm{B})$.

\section{Discussion}

Based on clinical characteristics, cytogenetic and molecular markers, more precise prognostic stratification has been established in AML and diagnosis and treatment individualization has become feasible [3]. The establishment of an effective monitoring method is essential for the evaluation of the early response and the adjustment of treatment regimens and the improvement of the prognosis as well.

MRD detection by flow cytometry has been applied to identify subclinical levels of leukemia cells and evaluate 

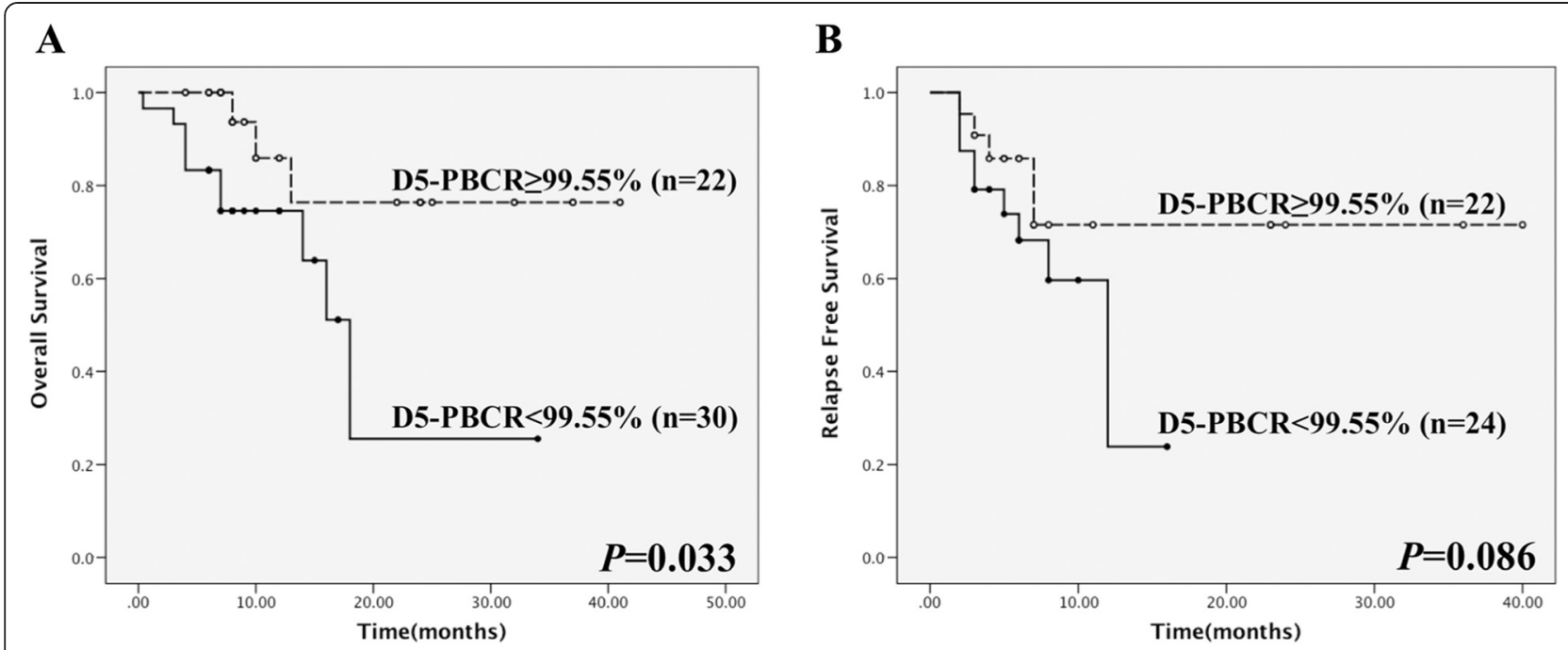

Figure 2 Survival analysis of patients in the cytogenetic-molecular intermediate-risk group. (A) OS of patients subdivided into high ( $\geq 99.55 \%$ ) and low (<99.55\%) D5-PBCR groups, $P=0.033$. (B) RFS of patients subdivided into high ( $\geq 99.55 \%)$ and low (<99.55\%) D5-PBCR groups, $P=0.086$.

treatment more precisely than conventional morphology; as such, this technique has been considered ideal for chemosensitivity assessment [6,17-21]. In ALL, MRD level $(<0.01 \%)$ and cytogenetic-molecular markers are both considered as independent outcome predictors [8]. MRD-based clinical approaches in children and adult ALL have yielded excellent results, which confirmed that MRD can be effective in risk stratification and treatment intervention [22-25].

In AML, MRD monitoring also plays an important role in the evaluation of treatment effect. Retrospective studies have demonstrated a high prognostic value of post-induction MRD level in AML. Terwijn et al. [26] and Freeman et al. [27] defined $0.1 \%$ as the MRD cut-off value; in both studies, MRD is correlated with RFS but not with OS. However, Inaba et al. [28] reported that MRD has a limited value in childhood AML if measured by MFC. Thus far, the threshold of MRD related to prognosis of AML remains controversial. MRD cannot be considered as an independent prognostic predictor in AML, in contrast to ALL [26,27,29].

Either in ALL or AML, the time points of BM-MRD monitoring are not earlier than 2 weeks after the induction begins. Although prognostic correlation is excellent, the time at which correlation is determined may be too late for early intervention of induction regimen. Thus,
$\mathbf{A}$

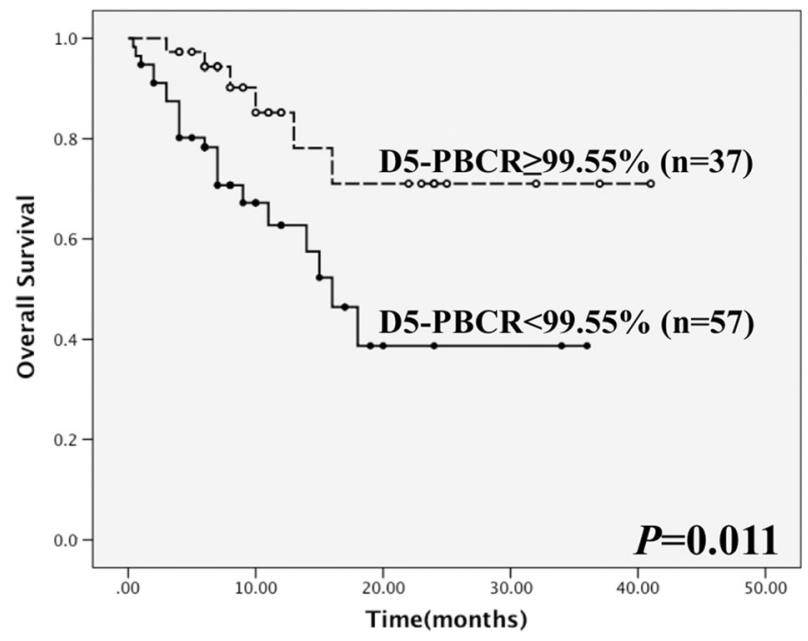

$\mathbf{B}$

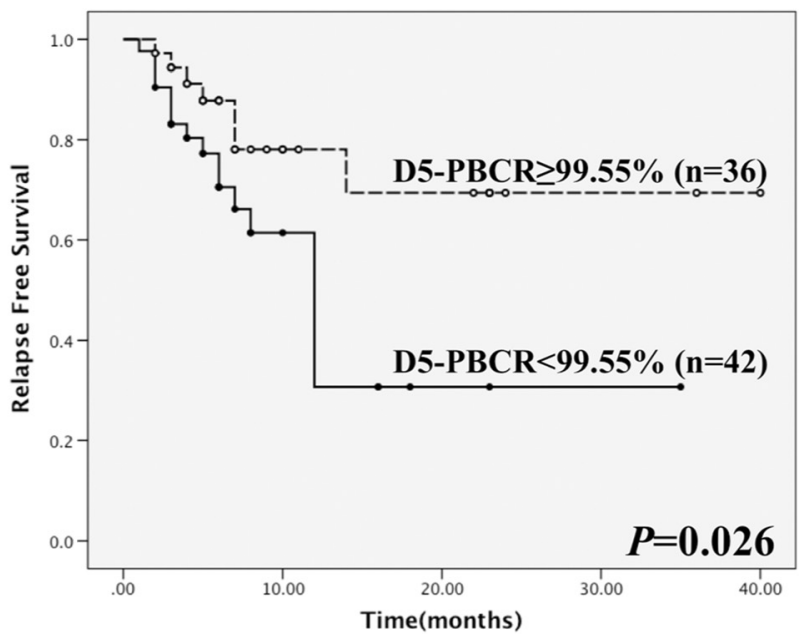

Figure 3 Survival analysis of whole cohort patients. (A) OS of patients subdivided into high ( $\geq 99.55 \%)$ and low (<99.55\%) D5-PBCR groups, $P=0.011$. (B) RFS of patients subdivided into high ( $299.55 \%)$ and low $(<99.55 \%)$ D5-PBCR groups, $P=0.026$. 
studies have evaluated peripheral blast clearance in the first week of induction.

In patients with ALL, practical values of PB blast assessment have been reported. Gajjar et al. [10] found that the persistence of circulating blasts after 1 week of therapy is significantly related to the worse event-free survival in childhood ALL. Atsushi et al. [11] also presented the same conclusion in patients with ALL treated with prednisolone monotherapy. Studies related to AML have also shown that a rapid decrease in peripheral leukemic burden determined through either morphology or flow cytometry $[12,15]$ is correlated with $\mathrm{CR}$ and longterm survival $[14,16]$.

In this study, we monitored the early treatment response of 96 AML patients by detecting PB blasts through flow cytometry. The PBRR at all checkpoints $\left(D_{3}-D_{9}\right)$ showed high prognostic value of $C R\left(D_{3}: P=0.005, D_{5}: P<\right.$ 0.0001, $\left.\mathrm{D}_{7}: P<0.0001, \mathrm{D}_{9}: P<0.0001\right)$. Starting from $\mathrm{D}_{5}$, PBRR was significantly related to the total relapse rates $\left(\mathrm{D}_{5}: P=0.005, \mathrm{D}_{7}: P=0.001, \mathrm{D}_{9}: P=0.0005\right)$ and early relapse rates $\left(\mathrm{D}_{5}: P=0.017, \mathrm{D}_{7}: P=0.008, \mathrm{D}_{9}: P=0.009\right)$. This finding is consistent with that in published studies in which $\mathrm{D}_{5}$ is also determined as the median time of $\mathrm{PB}$ blast clearance [12-16]. The ROC analysis identified D5PBCR of $99.55 \%$ initial blasts as the cut-off value. Patients with high D5-PBCR $(\geq 99.55 \%)$ showed greater CR rates and less relapse rates $(P<0.001, P=0.027)$ and were associated with significantly longer OS and RFS $(P=0.011$, $P=0.026)$.

Our D5-PBCR cut-off value might help optimize current cytogenetic-molecular prognostic risk stratification. In our cohort, $84.21 \%$ (16/19) of unfavorable-risk patients were classified into low D5-PBCR group (<99.55\%). In the intermediate-risk groups, D5-PBCR could further distinguish the subgroup of patients with relatively poorer prognosis, the two-year estimated OS and RFS rate were significantly worse in patients with D5-PBCR $<99.55 \%$ $(P=0.033, P=0.086)$, which were close to that of the unfavorable-risk group. This might facilitate further treatment regimen adjustments. The intermediate-risk patients with low D5-PBCR may be recommended for strengthening induction and consolidation therapy or receiving allo-HSCT.

$\mathrm{D}_{5}$ of induction is a valuable time point of early treatment response monitoring. This time point is appropriate to strengthen induction therapy. Augmented induction using dose-escalated regimens or three-drug combination has benefited patients [30-32]. Holowiecki et al. [31] also suggested that the addition of cladribine to the standard induction regimen can improve the outcome of patients with AML, particularly in the unfavorable-risk group. Thus, early risk evaluation by D5-PBCR provides the basis of individualized induction therapy.
The applicability of MRD by MFC is approximately $60 \%$ to $88 \%$ in patients with AML $[6,17]$. In patients with no LAIPs, BM MRD evaluation is unlikely performed through MFC assessment. In this case, D5-PBCR is considered as a new effective evaluation method. Almost all patients with AML suffer from circulating blasts at diagnosis, which enables $\mathrm{PB}$ blast monitoring a comparatively universal way. In our study, LAIP coverage was approximately $75 \%(72 / 96)$. Of the 24 patients with no LAIPs, 23 showed available data of D5-PBCR. The CR rate was $100 \%(7 / 7)$ in the high D5-PBCR group compared with $50 \%(8 / 16)$ in the low D5-PBCR group $(P=0.052)$; this result suggested that high D5-PBCR may help predict the CR status of patients without LAIPs.

However, for the few AML patients with very low percentage or even absent of $\mathrm{PB}$ blasts at diagnosis, BM MRD detection plays a more important role.

\section{Conclusions}

An effective evaluation method of early treatment response was established by monitoring PB blasts through MFC. D5-PBCR cut-off value might help distinguish high-risk patients in the first week of induction; thus, prognostic predictive ability of current risk stratification can be improved and induction regimen modification can be performed.

To further establish practical and precise clinical guidance, more patients need to be accumulated and multicenter confirmation is required.

\section{Methods}

\section{Patients and treatment protocols}

A total of 96 patients with de novo AML (non-M3) were enrolled in this study from June 2011 to August 2014 in the Shanghai Institute of Hematology. The diagnosis and classification of the AML subtypes were established according to FAB [33] and WHO 2008 criteria [34]. The Ethics Committee of Ruijin hospital approved this study. All patients provided informed consent according to the Declaration of Helsinki.

All patients received standard first-line chemotherapy. The induction regimen consisted of idarubicin (8 to 10 $\mathrm{mg} / \mathrm{m}^{2} / \mathrm{d}, \mathrm{D}_{1}-\mathrm{D}_{3}$ ) or daunorubicin (45 to $60 \mathrm{mg} / \mathrm{m}^{2} / \mathrm{d}$, $\left.D_{1}-D_{3}\right)$ and cytarabine $\left(100 \mathrm{mg} / \mathrm{m}^{2} / \mathrm{d}, \mathrm{D}_{1}-\mathrm{D}_{7}\right)$. After CR was achieved, consolidation therapy was administered according to the patients' cytogenetic-molecular risk stratification. Patients in favorable- and intermediaterisk group received HiDAC-based consolidation $\left(2 \mathrm{~g} / \mathrm{m}^{2}\right.$, every $12 \mathrm{~h}, \mathrm{D}_{1}-\mathrm{D}_{3}$ ), whereas patients in the unfavorablerisk group were recommended for allograft transplantation $(n=14)[30,35,36]$.

\section{Cytogenetic and molecular analysis}

Cytogenetic data was available in 88 of 96 cases. The chromosomes were R-banded and/or G-banded in unstimulated 
BM cells after $24 \mathrm{~h}$ of culture. The karyotype was analyzed according to the International System for Human Cytogenetic Nomenclature (2009) [37].

Molecular studies were performed in 94 cases. Mutations of several genes, including NPM1, NRAS, FLT3-ITD, FLT3-TKD, DNMT3A, CEBPA, MLL-PTD, C-KIT, were detected through RT-PCR and sequencing. $M L L$-related fusion genes, namely, MLL-AF9, MLL-AF10, MLL-AF6, $M L L-E L L, M L L-E N L$, and $M L L-A F 17$, were assessed through multiplex RT-PCR [36].

The cytogenetic-molecular risk groups were classified as follows: favorable group comprised $\mathrm{t}(8 ; 21), \mathrm{t}(16 ; 16)$ or inv (16), and normal cytogenetics with NPM1 mutation in the absence of FLT3-ITD, DNMT3A, and MLL-PTD mutations. Unfavorable group comprised $5 / 5 \mathrm{q}-,-7 / 7 \mathrm{q}-$, inv $(3) / \mathrm{t}(3 ; 3)$, t $(6 ; 9)$, complex karyotype ( $\geq 3$ clonal chromosomal abnormalities), and normal cytogenetics with FLT3-ITD. The intermediate group consisted of other patients [35].

\section{Identification of LAIPs}

Fresh $\mathrm{BM}$ or $\mathrm{PB}$ samples were processed according to the standard operating procedure of our institution [7]. The assays were performed using a 10-color flow cytometer (NAVIOS, Beckman Coulter, Brea, California, USA). Data were analyzed with KALUZA software (Beckman Coulter, Brea, California, USA). LAIPs were classified at diagnosis with different surface antigens (CD34, CD38, CD117, HLA-DR, CD13, CD33, CD14, CD15, CD64, CD2, CD4, CD7, CD11b, CD19, and CD56). The information on monoclonal antibody combination is shown in Additional file 4: Table S3.

More than $2.5 \times 10^{5}$ events from PB samples and $1 \times 10^{6}$ events from BM samples were required for flow cytometry detection to ensure sensitivity and accuracy. For BM samples, a cut-off value of $0.1 \%$ was determined as MRD positive.

\section{Calculation of PBRR and PBCR}

PB samples of right before treatment $\left(D_{0}\right)$ and on the 3rd, 5th, 7th, 9th days $\left(D_{3}, 5,7,9\right)$ of the induction chemotherapy were collected. The absolute counts of blasts in PB were calculated using flow count fluorospheres (Beckman Coulter, Brea, California, USA). For patients with LAIPs, blasts with the specific immunophenotype were counted. For patients with no LAIPs, PB blasts were determined with surface antigens (including CD34+, CD117+, CD33+, CD13+, HLA-DR+, and CD45dim) associated with myeloid lineage cells. Patients whose $\mathrm{PB}$ blast percentage was higher than $0.5 \%$ at $D_{0}$ were included in this study. PBRR was defined as the logarithmic ratio to the absolute counts of $D_{0}\left[\log _{10}\left(D_{0} / D_{x}\right)\right]$ [38]. PBCR was defined as the percentage of absolute count reduction: $\left[\left(D_{0}-D_{x}\right) / D_{0} \times 100 \%\right]$.

\section{Statistical analysis}

PBCRs were compared through independent-sample $t$ test. An ROC analysis was performed to evaluate the predictive efficiency of PBRR on patients' CR. Statistical significance was considered when AUC was $>0.6$; the cutoff value was selected according to the maximum sum of sensitivity and specificity [39]. Chi-square test was performed to compare CR and relapse rates. OS was defined as the time of diagnosis to death or allo-HSCT or the last follow-up. RFS was defined as the time at which CR was achieved until relapse occurred and was also defined on the basis of whether a patient died or remained alive when CR was achieved at the last follow-up (censored). OS and RFS were estimated by using Kaplan-Meier method and compared by conducting log-rank test. The last follow-up was performed in December 2014, and the median followup time was $11(0.4-41)$ months. Two-sided $P<0.05$ was considered statistically significant. Statistical analyses were evaluated using SPSS 22.0 software (IBM, Armonk, NY, USA).

\section{Additional files}

\begin{abstract}
Additional file 1: Table S1. LAIP characteristics of patients. LAIPS were identified in 72 patients with four main types: 63 cases with cross-lineage antigen expression, 6 cases with asynchronous antigen expression, 13 cases with antigen dim/strong expression, and 8 cases with antigen expression missing.
\end{abstract}

Additional file 2: Table S2. Gene mutation profile of cytogenetic-normal AML patients stratified by D5-PBCR. Mutations of several genes, including NRAS, NPM1, FLT3-ITD, FLT3-TKD, DNMT3A, CEBPA, MLL-PTD, C-KIT, and MLLrelated fusion genes were detected in 58 cytogenetic-normal AML patients, 57 showed available data of D5-PBCR. A trend of more NRAS (14.8\% vs. $0 \%$, $P=0.051)$, CEBPA biallelic mutations (37.0\% vs. $20.7 \%, P=0.142)$, and less MLL-PTD mutations ( $3 \times .7 \%$ vs. $10.3 \%, P=0.612$ ) was observed in the D5-PBCR $\geq 99.55 \%$ group.

Additional file 3: Figure S1. Survival analysis of patients with cytogenetic-molecular risk stratification. (A) OS of patients subdivided into favorable-risk group, intermediate-risk group with high D5-PBCR ( $\geq 99.55 \%)$, intermediate-risk group with low D5-PBCR (<99.55\%), and unfavorable-risk group, $P<0.001$. (B) RFS of patients subdivided into favorable-risk group, intermediate-risk group with high D5-PBCR ( $\geq 99.55 \%)$, intermediate-risk group with low D5-PBCR (<99.55\%), and unfavorable-risk group, $P=0.010$.

Additional file 4: Table S3. The mAbs combinations utilized for MRD and PB blast assessment. The monoclonal antibody combination utilized for MRD and PB blast assessment included seven fixed mAbs (CD34, HLA-DR, CD13, CD33, CD117, CD10, and CD45) and eight alternative mAbs (CD2, CD4, CD7, CD19, CD56, CD11b, CD64, and CD14).

\section{Abbreviations}

ALL: acute lymphoblastic leukemia; allo-HSCT: allogeneic hematopoietic cell transplantation; AUC: area under the curve; AML: acute myeloid leukemia; BM: bone marrow; CR: complete remission; FAB: French-American-British; LAIP: leukemia-associated aberrant immunophenotype; MRD: minimal residual disease; MFC: multiparameter flow cytometry; PB: peripheral blood; NCR: not achieved complete remission; OS: overall survival; PBCR: peripheral blasts clearance rate; PBRR: PB blasts reduction ratio; RFS: relapse-free survival; ROC: receiver operating characteristic.

\section{Competing interests}

The authors declare that they have no competing interests. 


\section{Authors' contributions}

CSJ and CB designed the research. YC and KQL performed the experiments. ZYX and CB analyzed data. WXQ, WJ, SY, JCL, ZYM, CQ, XSM and LJM provided technical and clinical expertise. CB wrote the manuscript. CSJ and XXD edited the manuscript. All authors have read and approved the final manuscript.

\section{Acknowledgements}

This work was supported in part by the National High Tech Program for Biotechnology Grant 863 (2012AA02A505), the National Natural Science Foundation of China (81470311, 81270619, 81123005), the Ministry of Health Grant (201202003), and the Mega-projects of Scientific Research for the 12th Five-Year Plan Grant (2013ZX09303302).

\section{Received: 12 February 2015 Accepted: 28 April 2015 Published online: 10 May 2015}

\section{References}

1. Duque-Afonso J, Cleary ML. The AML salad bowl. Cancer Cell. 2014;25(3):265-7. doi:10.1016/j.ccr.2014.03.002.

2. Cancer Genome Atlas Research N. Genomic and epigenomic landscapes of adult de novo acute myeloid leukemia. N Engl J Med. 2013;368(22):2059-74. doi:10.1056/NEJMoa1301689.

3. Estey EH. Acute myeloid leukemia: 2014 update on risk-stratification and management. Am J Hematol. 2014;89(11):1063-81. doi:10.1002/ajh.23834

4. Pulte D, Redaniel MT, Jansen L, Brenner H, Jeffreys M. Recent trends in survival of adult patients with acute leukemia: overall improvements, but persistent and partly increasing disparity in survival of patients from minority groups. Haematologica. 2013;98(2):222-9. doi:10.3324/haematol.2012.063602.

5. Cornelissen JJ, Versluis J, Passweg JR, van Putten WL, Manz MG, Maertens J, et al. Comparative therapeutic value of post remission approaches in patients with acute myeloid leukemia aged 40-60 years. Leukemia. 2014. doi:10.1038/leu.2014.332

6. Al-Mawali A, Gillis D, Lewis I. The role of multiparameter flow cytometry for detection of minimal residual disease in acute myeloid leukemia. Am J Clin Pathol. 2009;131(1):16-26. doi:10.1309/AJCP5TSD3DZXFLCX.

7. Weng XQ, Shen $Y$, Sheng $Y$, Chen $B$, Wang JH, Li JM, et al. Prognostic significance of monitoring leukemia-associated immunophenotypes by eight-color flow cytometry in adult B-acute lymphoblastic leukemia. Blood Cancer J. 2013;3:e133. doi:10.1038/bcj.2013.31.

8. Beldjord K, Chevret S, Asnafi V, Huguet F, Boulland ML, Leguay T, et al. Oncogenetics and minimal residual disease are independent outcome predictors in adult patients with acute lymphoblastic leukemia. Blood. 2014;123(24):3739-49. doi:10.1182/blood-2014-01-547695.

9. Paietta E. When it comes to MRD, AML not equal ALL. Blood. 2012;120(8):1536-7. doi:10.1182/blood-2012-06-435081.

10. Gajjar A, Ribeiro R, Hancock ML, Rivera GK, Mahmoud H, Sandlund JT, et al. Persistence of circulating blasts after 1 week of multiagent chemotherapy confers a poor prognosis in childhood acute lymphoblastic leukemia. Blood. 1995;86(4):1292-5.

11. Manabe A, Ohara A, Hasegawa D, Koh K, Saito T, Kiyokawa N, et al. Significance of the complete clearance of peripheral blasts after 7 days of prednisolone treatment in children with acute lymphoblastic leukemia: the Tokyo children's cancer study group study L99-15. Haematologica. 2008;93(8):1155-60. doi:10.3324/haematol.12365.

12. Gianfaldoni G, Mannelli F, Baccini M, Antonioli E, Leoni F, Bosi A. Clearance of leukaemic blasts from peripheral blood during standard induction treatment predicts the bone marrow response in acute myeloid leukaemia: a pilot study. Br J Haematol. 2006;134(1):54-7. doi:10.1111/j.1365-2141.2006.06100.x.

13. Elliott MA, Litzow MR, Letendre LL, Wolf RC, Hanson CA, Tefferi A, et al. Early peripheral blood blast clearance during induction chemotherapy for acute myeloid leukemia predicts superior relapse-free survival. Blood. 2007;110(13):4172-4. doi:10.1182/blood-2007-07-104091.

14. Arellano M, Pakkala S, Langston A, Tighiouart M, Pan L, Chen Z, et al. Early clearance of peripheral blood blasts predicts response to induction chemotherapy in acute myeloid leukemia. Cancer. 2012;118(21):5278-82. doi:10.1002/cncr.27494

15. Lacombe F, Arnoulet C, Maynadie M, Lippert E, Luquet I, Pigneux A, et al Early clearance of peripheral blasts measured by flow cytometry during the first week of AML induction therapy as a new independent prognostic factor: a GOELAMS study. Leukemia. 2009;23(2):350-7. doi:10.1038/leu.2008.296.

16. San Miguel JF, Vidriales MB, Lopez-Berges C, Diaz-Mediavilla J, Gutierrez N, Canizo C, et al. Early immunophenotypical evaluation of minimal residual disease in acute myeloid leukemia identifies different patient risk groups and may contribute to postinduction treatment stratification. Blood. 2001;98(6):1746-51.

17. Hourigan CS, Karp JE. Minimal residual disease in acute myeloid leukaemia. Nat Rev Clin Oncol. 2013;10(8):460-71. doi:10.1038/nrclinonc.2013.100.

18. Al-Mawali A, Gillis D, Hissaria P, Lewis I. Incidence, sensitivity, and specificity of leukemia-associated phenotypes in acute myeloid leukemia using specific five-color multiparameter flow cytometry. Am J Clin Pathol. 2008;129(6):934-45. doi:10.1309/FYOUMAMM91VPMR2W.

19. Ossenkoppele G, Schuurhuis GJ. MRD in AML: time for redefinition of CR? Blood. 2013;121(12):2166-8. doi:10.1182/blood-2013-01-480590.

20. Ossenkoppele GJ, Schuurhuis GJ. MRD in AML: it is time to change the definition of remission. Best Pract Res Clin Haematol. 2014;27(3-4):265-71. doi:10.1016/j.beha.2014.10.008.

21. Grimwade D, Freeman SD. Defining minimal residual disease in acute myeloid leukemia: which platforms are ready for "prime time"? Blood. 2014;124(23):3345-55. doi:10.1182/blood-2014-05-577593.

22. Roberts KG, Pei D, Campana D, Payne-Turner D, Li Y, Cheng C, et al. Outcomes of children with bcr-abl1-like acute lymphoblastic leukemia treated with risk-directed therapy based on the levels of minimal residual disease. J Clin Oncol. 2014. doi:10.1200/JCO.2014.55.4105

23. Ribera JM, Oriol A, Morgades M, Montesinos P, Sarra J, Gonzalez-Campos J, et al. Treatment of high-risk Philadelphia chromosome-negative acute lymphoblastic leukemia in adolescents and adults according to early cytologic response and minimal residual disease after consolidation assessed by flow cytometry: final results of the PETHEMA ALL-AR-03 trial. J Clin Oncol. 2014;32(15):1595-604. doi:10.1200/JCO.2013.52.2425.

24. Vora A, Goulden N, Mitchell C, Hancock J, Hough R, Rowntree C. Augmented post-remission therapy for a minimal residual disease-defined high-risk subgroup of children and young people with clinical standard-risk and intermediate-risk acute lymphoblastic leukaemia (UKALL 2003): a randomised controlled trial. Lancet Oncol. 15;8:809-18. doi:10.1016/s14702045(14)70243-8.

25. Salari F, Shahjahani M, Shahrabi S, Saki N. Minimal residual disease in acute lymphoblastic leukemia: optimal methods and clinical relevance, pitfalls and recent approaches. Med Oncol. 2014;31(11):266. doi:10.1007/s12032-014-0266-3.

26. Terwijn M, van Putten WL, Kelder A, van der Velden VH, Brooimans RA, Pabst T, et al. High prognostic impact of flow cytometric minimal residual disease detection in acute myeloid leukemia: data from the HOVON/SAKK AML 42A study. J Clin Oncol. 2013;31(31):3889-97. doi:10.1200/JCO.2012.45.9628

27. Freeman SD, Virgo P, Couzens S, Grimwade D, Russell N, Hills RK, et al. Prognostic relevance of treatment response measured by flow cytometric residual disease detection in older patients with acute myeloid leukemia. J Clin Oncol. 2013;31(32):4123-31. doi:10.1200/JCO.2013.49.1753

28. Inaba H, Coustan-Smith E, Cao X, Pounds SB, Shurtleff SA, Wang KY, et al. Comparative analysis of different approaches to measure treatment response in acute myeloid leukemia. J Clin Oncol. 2012;30(29):3625-32. doi:10.1200/JCO.2011.41.5323.

29. Kohnke T, Sauter D, Ringel K, Hoster E, Laubender RP, Hubmann M, et al. Early assessment of minimal residual disease in AML by flow cytometry during aplasia identifies patients at increased risk of relapse. Leukemia. 2015;29(2):377-86. doi:10.1038/leu.2014.186.

30. Jin J, Wang J-X, Chen F-F, Wu D-P, Hu J, Zhou J-F, et al. Homoharringtonine-based induction regimens for patients with de-novo acute myeloid leukaemia: a multicentre, open-label, randomised, controlled phase 3 trial. Lancet Oncol. 2013;14(7):599-608. doi:10.1016/s1470-2045(13)70152-9.

31. Holowiecki J, Grosicki S, Giebel S, Robak T, Kyrcz-Krzemien S, Kuliczkowski K, et al. Cladribine, but not fludarabine, added to daunorubicin and cytarabine during induction prolongs survival of patients with acute myeloid leukemia: a multicenter, randomized phase III study. J Clin Oncol. 2012;30(20):2441-8. doi:10.1200/JCO.2011.37.1286.

32. Fernandez HF, Sun Z, Yao X, Litzow MR, Luger SM, Paietta EM, et al. Anthracycline dose intensification in acute myeloid leukemia. N Engl J Med. 2009;361(13):1249-59. doi:10.1056/NEJMoa0904544. 
33. Bennett JM, Catovsky D, Daniel MT, Flandrin G, Galton DA, Gralnick HR, et al. Proposals for the classification of the acute leukaemias. French-American-British (FAB) Co-operative group. Br J Haematol. 1976;33(4):451-8.

34. Swerdlow SH, Campo E, Harris NL, Jaffe ES, Pileri SA, Stein H, et al. WHO classification of tumours of haematopoietic and lymphoid tissues. 4th ed. International Agency for Research on Cancer; 2008.

35. Network NCC. NCCN Guidelines: acute myeloid leukemia version 2.2014 2014

36. Shen Y, Zhu YM, Fan X, Shi JY, Wang QR, Yan XJ, et al. Gene mutation patterns and their prognostic impact in a cohort of 1185 patients with acute myeloid leukemia. Blood. 2011;118(20):5593-603. doi:10.1182/blood-2011-03-343988.

37. Chia NL. A comprehensive set of idiograms representing all interpretive levels of resolution: ISCN (2009). Cytogenet Genome Res. 2009;125(2):162-4. doi:10.1159/000227842

38. Rossi G, Minervini MM, Melillo L, di Nardo F, de Waure C, Scalzulli PR, et al. Predictive role of minimal residual disease and log clearance in acute myeloid leukemia: a comparison between multiparameter flow cytometry and Wilm's tumor 1 levels. Ann Hematol. 2014;93(7):1149-57. doi:10.1007/s00277-014-2029-9

39. Fawcett T. An introduction to ROC analysis. Pattern Recogn Lett. 2006;27(8):861-74. doi:10.1016/j.patrec.2005.10.010.

\section{Submit your next manuscript to BioMed Central and take full advantage of:}

- Convenient online submission

- Thorough peer review

- No space constraints or color figure charges

- Immediate publication on acceptance

- Inclusion in PubMed, CAS, Scopus and Google Scholar

- Research which is freely available for redistribution 